\title{
Atividade moluscicida frente ao caramujo Biomphalaria glabrata do óleo essencial de
}

\section{Origanum vulgare $\mathbf{L}$.}

\author{
Molluscicidal activity in front of the Biomphalaria glabrata snail of the essential oil of Origanum \\ vulgare $\mathbf{L}$. \\ Actividad moluscicida frente al caracol Biomphalaria glabrata del aceite esencial de Origanum \\ vulgare $\mathbf{L}$.
}

Thércia Gabrielle Teixeira Martins ORCID: https://orcid.org/0000-0002-0506-1748

Universidade Federal do Maranhão, Brasil

E-mail: thercia.martins@ @otmail.com

Ana Patrícia Matos Pereira

ORCID: https://orcid.org/0000-0003-4478-4209

Universidade Federal do Maranhão, Brasil

E-mail: ap.matos11@hotmail.com

Paulo Victor Serra Rosa

ORCID: https://orcid.org/0000-0003-1782-5896

Universidade Federal de Santa Catarina, Brasil

E-mail: paullovictorserra@gmail.com

Leila da Silva Silveira

ORCID: https://orcid.org/0000-0003-1050-4483

Universidade Federal do Maranhão, Brasil

E-mail: alielarcanjo@hotmail.com

Francilidia Oliveira Vitorino de Assunção Conceição

ORCID: https://orcid.org/0000-0002-0079-7502

Universidade Federal do Maranhão, Brasil

E-mail: francilidiaassuncao@gmail.com

Carlos Eduardo Pereira Conceição

ORCID: https://orcid.org/0000-0002-6735-9291 Hospital Guarás, Brasil

E-mail: dudaecarol21@gmail.com

Thayson Rodrigues Lopes

ORCID: https://orcid.org/0000-0002-5112-4658 Secretaria de Educação do Estado do Maranhão, Brasil

E-mail: thaysonphb@gmail.com

Danielly Fonseca

ORCID: https://orcid.org/0000-0003-1782-5896

Universidade Federal de Santa Catarina, Brasil

E-mail: danyfonseca2002@gmail.com

Nilgicy Maria de Jesus Amorim

ORCID: https://orcid.org/0000-0001-9473-6470

Hospital Universitário Presidente Dutra, Brasil

E-mail: nilgicy@gmail.com

Christyann Lima Campos Batista

ORCID: https://orcid.org/0000-0001-5431-5620

Universidade de São Paulo, Brasil

E-mail: chrisbatista@usp.br

Ari Pereira de Araújo Neto

ORCID: https://orcid.org/0000-0001-6903-4127

Universidade Federal do Delta do Parnaíba, Brasil

E-mail: aripereiraneto@gmail.com

Gustavo Oliveira Everton

ORCID: https://orcid.org/0000-0002-0457-914X

Universidade Federal do Maranhão, Brasil

E-mail: gustavooliveiraeverton@gmail.com

\section{Resumo}

Este estudo avaliou a atividade moluscicida frente ao caramujo transmissor da esquistossomose (Biomphalaria glabrata) do óleo essencial de Origanum vulgare. O material vegetal foi coletado no munícipio de São Luis (MA). O 
óleo essencial foi extraído por hidrodestilação a $100^{\circ} \mathrm{C}$ por 3h, com caracterização química através de Cromatografia Gasosa acoplada a Espectrometria de Massas (CG-EM). Para atividade moluscicida executou-se a metodologia preconizada pela OMS, sendo a $\mathrm{CL}_{50}$ do óleo essencial para ação dos mesmos frente ao caramujo obtido pelo método de Probit. Os constituintes majoritários encontrados no óleo essencial de $O$. vulgare foram Timol $(72,14 \%)$ e pCimeno (12,25\%). A atividade moluscicida do óleo essencial apresentou a $\mathrm{CL}_{50}$ de $26,55 \mathrm{mg} \mathrm{L}^{-1}$. Os resultados indicam que o óleo essencial avaliado é composto por substâncias que propiciam e incentivam sua aplicação, devido ao seu alto potencial para atividade moluscicida frente a Biomphalaria glabrata.

Palavras-chave: Bioproduto; Moluscicida; Óleo essencial; Origanum vulgare.

\begin{abstract}
This study evaluated the molluscicidal activity in front of the snail transmitting the schistosomes (Biomphalaria glabrata) of the essential oil of Origanum vulgare. The plant material was collected in the municipality of São Luis (MA). The essential oil was extracted by hydrodistillation at $100^{\circ} \mathrm{C}$ for $3 \mathrm{~h}$, with chemical characterization through Gas Chromatography coupled to Mass Spectrometry (GC-MS). For molluscicidal activity, the methodology recommended by the WHO was performed, and the $\mathrm{LC}_{50}$ of the essential oil was used in the face of the snail obtained by the Probit method. The major constituents found in the essential oil of $O$. vulgare were Timol $(72,14 \%)$ and $\mathrm{p}$ cimene $(12,25 \%)$. A atividade moluscicida do óleo essencial apresentou a $\mathrm{CL}_{50}$ de $26,55 \mathrm{mg} \mathrm{L}^{-1}$. The results indicate that the evaluated essential oil is composed of substances that promote and encourage its application, due to its high potential for molluscicidal activity against Biomphalaria glabrata.
\end{abstract}

Keywords: Bioproduct; Molluscicide; Essential oil; Origanum vulgare.

\title{
Resumen
}

Este estudio evaluó la actividad moluscicida frente al caracol transmitiendo los esquistosomas (Biomphalaria glabrata) del aceite esencial de Origanum vulgare. El material vegetal fue recolectado en el municipio de São Luis (MA). El aceite esencial se extrajo por hidrodestilación a $100^{\circ} \mathrm{C}$ durante $3 \mathrm{~h}$, con caracterización química mediante Cromatografía de Gases acoplada a Espectrometría de Masas (GC-MS). Para la actividad moluscicida se realizó la metodología recomendada por la OMS, y se utilizó la $\mathrm{LC}_{50}$ del aceite esencial frente al caracol obtenido por el método Probit. Los principales componentes encontrados en el aceite esencial de $O$. vulgare fueron Timol $(72,14 \%)$ y pcimene $(12,25 \%)$. La actividad moluscicida del aceite esencial presentó la $\mathrm{LC}_{50}$ de $26,55 \mathrm{mg} \mathrm{L}^{-1}$. Los resultados indican que el aceite esencial evaluado está compuesto por sustancias que proporcionan y fomentan su aplicación, debido a su alto potencial de actividad moluscicida contra Biomphalaria glabrata.

Palabras clave: Bioproducto; Moluscicida; Aceite esencial; Origanum vulgare.

\section{Introdução}

A esquistossomose é uma doença endêmica em vários países tropicais, causada pelo Schistosoma mansoni. Esta moléstia é umas das mais prevalentes infecções parasitárias observada nos países tropicais, impactando negativamente a economia e principalmente a saúde pública. Os hospedeiros intermediários do S. mansoni são espécies pertencentes aos gêneros Biomphalaria, Bulinus e Oncomelania (Sá et al., 2017). No Brasil, os hospedeiros intermediários pertecem ao gênero Biomphalaria (Gomes et al, 2019).

B. glabrata é um caramujo da família Planorbidae, que pode ser encontrado em vários países, nos ambientes aquáticos com água parada ou pouca corrente, tais como lagos, lagoas, poços, remansos de rios, riachos, canais de irrigação e de drenagem, e qualquer área natural ou artificialmente alagada (Stensgaard et al., 2013; Mccreesh \& Booth, 2014; Sá et al., 2017).

A presença de esquistossomose é indicadora de condições precárias e este enfoque deve estar presente na mente de todos os especialistas no trabalho de controle desta endemia. O controle da esquistossomose demanda medidas que englobam tratamento de portadores, saneamento básico, educação sanitária e aplicação de moluscicidas (Everton et al., 2018).

As substâncias moluscicida são empregadas para o extermínio de moluscos e utilizadas para controlar caramujos vetores de parasitas. Dentre os moluscicidas sintéticos, os mais utilizados são: hidróxido de cálcio, Frscon, Gramaxone, Bayluscid (niclosamida) (Catanhede et al., 2010). E a problemática no uso desses sintéticos químicos é a toxicidade para os organismos não-alvo, contaminação ambiental e resistências dos moluscos da espécie B. glabrata. Surge então a necessidade de buscar a utilização de moluscicidas de origem vegetal. 
Conforme Luna et al. (2006), a esquistossomose se enquadra nas chamadas doenças negligenciável, portanto, os estudos com plantas com propriedades moluscicidas, que sejam de fácil acesso à população, representa uma forma de redução do risco de transmissão da esquistossomose, pois age diretamente no ciclo da doença e constitui-se uma alternativa de baixo custo.

Nos últimos anos, ocorre uma atenção considerável aos efeitos biológicos de produtos obtidos de fontes vegetais, entre eles, os óleos essenciais (OEs) obtidos de plantas aromáticas e seus componentes (Júnior et al., 2020). Os óleos essenciais (OEs) vêm ganhando grande espaço por apresentar diversas aplicações, como no uso contra agentes patológicos, além de ser uma opção com menor toxicidade em comparação a outros produtos de natureza sintética (Miranda et al., 2016). Os OEs apresentam uma composição relativamente complexa, podendo possuir entre dezenas a centenas de compostos, e em geral o fitoconstituinte em maior concentração é aquele que atribui à atividade biológica do OE (Lubbe \& Verpoorte, 2007; Cunha, 2012).

Dentre as plantas produtoras de óleo essencial destaca-se Origanum vulgare L., pertence à família Lamiaceae, seu óleo essencial é composto predominantemente por fenóis, como carvacrol e timol (Silva et al., 2020). Desta forma, o presente estudo tem por objetivo determinar os constituintes químicos e atividade moluscicida frente ao caramujo transmissor da esquistossomose do óleo essencial de Origanum vulgare.

\section{Metodologia}

\subsection{Material vegetal}

A coleta do material vegetal utilizado nesta pesquisa foi realizada de outubro a dezembro de 2019. Folhas de $O$. vulgare foram coletadas no município de São Luís, Brasil. As amostras foram depositadas no Herbário Ático Seabra da Universidade Federal do Maranhão. Após a coleta, as espécies vegetais foram transportadas para o Laboratório de Pesquisa e Aplicação de Óleos Essenciais (LOEPAV/UFMA).

\subsection{Obtenção do óleo essencial}

Para a extração do $\mathrm{OE}$, a técnica de foi utilizada com um extrator de vidro Clevenger acoplado a um balão de fundo redondo acoplado a uma manta elétrica como fonte geradora de calor. Foram utilizados $100 \mathrm{~g}$ do material vegetal, adicionando água destilada (1:10). A hidrodestilação foi realizada a $100^{\circ} \mathrm{C}$ durante $3 \mathrm{~h}$ e o OE extraído foi coletado. Cada OE foi seco por percolação com sulfato de sódio anidro $\left(\mathrm{Na}_{2} \mathrm{SO}_{4}\right)$. Essas operações foram realizadas em triplicados e as amostras armazenadas em ampolas de vidro âmbar sob refrigeração de $4^{\circ} \mathrm{C}$. Posteriormente submetidas às análises.

\subsection{Análises de constituintes químicos}

Os constituintes dos OEs foram identificados por cromatografia gasosa acoplado à espectrometria de massa (GC-MS). $1,0 \mathrm{mg}$ da amostra foi dissolvida em $1000 \mu \mathrm{L}$ de diclorometano (pureza 99,9\%). O programa AMDIS (Automated Mass Spectral Deconvolution Mass \& Identification System) foi utilizado para identificar os compostos da amostra.

\subsection{Atividade moluscicida}

Para a avaliação da atividade moluscicida, utilizou-se a técnica recomendada pela Organização Mundial da Saúde (1983), onde foram realizados dois testes em triplicata. No primeiro, chamado de teste piloto, uma solução do óleo em estudo foi preparada em um volume de $500 \mathrm{~mL}$ a uma concentração de $100 \mathrm{mg} \mathrm{L}^{-1}$ e Tween 80-tensoativo (2\%), onde 10 caramujos adultos foram colocados, negativos para Schistossoma mansoni, obtendo-se no final uma razão de $50 \mathrm{~mL} /$ caramujo e alimentando-os com alface hidropônica. 
Foram expostos na solução por 24h, à temperatura ambiente, retirados da solução, lavados duas vezes com água destilada desclorada, colocados em um recipiente de vidro contendo $500 \mathrm{~mL}$ de água destilada desclorada, alimentando-as com alface hidropônica e observada a cada 24 horas durante 4 dias para avaliar a mortalidade.

No segundo teste, chamado de concentração letal $\left(\mathrm{CL}_{50}\right)$, foram preparadas soluções de cada óleo em um volume de $500 \mathrm{~mL}$ nas concentrações $100,75,62,5,50,20,10,5$ e $2 \mathrm{mg} \mathrm{L}^{-1}$ e Tween 80 (2\%), utilizando a mesma metodologia do teste piloto. Para o controle negativo, também foram utilizados dois testes, no primeiro colocamos $500 \mathrm{~mL}$ de água desclorada e 10 caramujos em um recipiente de vidro e no segundo 10 caramujos imersos em uma solução com Tween 80 (2\%) em $500 \mathrm{~mL}$ de água destilada, alimentando-se tanto com alface hidropônica quanto a análise também realizada nos testes anteriores.

As taxas de mortalidade foram obtidas pela média de indivíduos mortos em função do logaritmo da dose testada. A análise estatística dos dados para o $\mathrm{CL}_{50}$ foi realizada de acordo com o método Probit. A concentração letal $\mathrm{CL}_{90}$ do bioensaio foi determinada pela regressão linear, obtendo a razão concentração versus mortalidade dos moluscos (Colegate\&Molyneux, 2007).

\section{Resultados e Discussão}

\subsection{Constituintes químicos}

De acordo com os resultados obtidos através da Cromatografia Gasosa acoplada a Espectrometria de Massas (CG/EM) o composto majoritário do óleo essencial das folhas de Origanum vulgare L. foi o timol (72,14\%), seguido do pcimeno $(12,25 \%)$.

De acordo com o cromatograma obtido da amostra do óleo essencial de orégano (O. vulgare L.) analisada, pode-se constatar como metabólitos secundários majoritários, timol (72,14\%), seguido do p-cimeno (12,25\%).

De modo semelhante, em estudo de Sarikurkcu et al., (2015) também encontraram através da Cromatografia Gasosa acoplada à Espectrometria de Massas (CG/EM), o timol (58,31\%) e $p$ - cimeno (13,45\%), como compostos majoritários do OE de $O$. vulgare extraído na Turquia. La Pergola et al., (2017), relataram o timol e $\gamma$-terpineno com 48,7 e 20,5\%, respectivamente, da amostra de $O$. vulgare subsp. hirtum coletado em Sicília.

Em outro estudo proposto por Khan et al., (2018), avaliaram a composição química das partes aéreas do OE de $O$. vulgare onde encontraram carvacrol (70,2 $\pm 1,37 \%), y$-terpineno $(5,6 \pm 0,11 \%)$, p-cimeno $(4,5 \pm 0,42 \%)$, hidrato de transsabineno $(3,8 \pm 0,07 \%)$ e timol $(2,2 \pm 0,12 \%)$. Outros autores também relatam como constituintes majoritários o $\alpha$-terpineno, $\gamma$ terpineno, linalol, 4-terpineol, timol, assim como o p-cimeno (Blank et al., 2016; Borges et al., 2012).

Fatores ambientais como a sazonalidade, temperatura, local de coleta, solo, disponibilidade de água, vento, latitude, além de modo de extração, rendimento e outros fatores, podem influenciar de forma significativa na composição química de óleos essenciais (Silva\&Heinzmann,2018; Ribeiro; Bonilla; Lucena, 2018), o que justifica a variação entre os compostos químicos encontrados em outros estudos em relação com os resultados desta pesquisa.

\subsection{Atividade moluscicida}

Na Tabela 1 são apresentados os resultados referentes a atividade moluscicida do OE de $O$. vulgare L. frente ao Biomphalaria glabrata. Apesar de haver muitos estudos relacionados a produtos naturais do gênero Origanum, não há relatos na literatura sobre o uso de óleos essenciais da espécie $O$. vulgare L. (orégano) como agente moluscicida, sendo assim, o presente estudo tem caráter inédito para essa espécie. 
Tabela 1 - Mortalidade Biomphalaria glabrata para ação do óleo essencial pelo método Probit.

\begin{tabular}{|c|c|c|c|c|c|c|}
\hline $\log C$ & Mortality & $\begin{array}{l}\mathrm{CL}_{50} \\
\mathrm{mg} \mathrm{L}^{-1}\end{array}$ & $\begin{array}{l}\mathrm{CL}_{90} \\
\mathrm{mg} \mathrm{L}^{-1}\end{array}$ & $\chi^{2}$ & $\boldsymbol{\sigma}$ & $\mathbf{R}^{2}$ \\
\hline 1,00 & 26,5 & & & & & \\
\hline 1,30 & 37,2 & & & & & \\
\hline 1,48 & 48,0 & & & & & \\
\hline 1,70 & 56,8 & & & & & \\
\hline 1,78 & 75,5 & 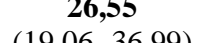 & $\begin{array}{c}\mathbf{8 7 , 8 7} \\
\end{array}$ & 0,998 & 0,489 & 0,932 \\
\hline 1,85 & 85,3 & & & & & \\
\hline 1,95 & 85,3 & & & & & \\
\hline 2,00 & 90,2 & & & & & \\
\hline 2,08 & 93,1 & & & & & \\
\hline
\end{tabular}

Fonte: Autores.

De acordo com a Tabela 1, o OE de $O$. vulgare L. obteve uma Concentração Letal 50\% (CL50) de 26,55 mg L-1, sendo relativamente baixa e $\mathrm{CL}_{90}$ de $87,87 \mathrm{mg} \mathrm{L}^{-1}$. Esses resultados estão de acordo com os critérios estabelecidos pela OMS (1983), que estabelece que espécies vegetais tem potencial moluscicida ativo em concentrações de até $100 \mathrm{mg} \mathrm{L}^{-1}$.

Estes resultados tornam-se satisfatórios quando comparados aos de Ribeiro (2016) que avaliou o efeito moluscicida do OE de Hyptis dilatata Benth, pertencente à família da Lamiaceae, sendo a mesma do O. vulgare, frente ao Biomphalaria glabrata e obteve as $\mathrm{CL}_{50}$ e $\mathrm{CL}_{90}$ de $112,46 \mu \mathrm{g} \mathrm{mL}^{-1}$ e $182,33 \mu \mathrm{g} \mathrm{mL}^{-1}$, considerados fora dos valores normatizados pela OMS.

Para uma substância ser classificada com ação moluscicida, deve eliminar o caramujo em todas as fases de sua vida e no seu habitat natural, com baixo custo, baixas concentrações, não apresentar riscos ao ser humano e ao ambiente, ser de fácil aplicação, além de apresentar estabilidade frente a mudanças de temperatura e irradiação solar (OMS,1983). Assim, O OE em estudo está dentro desses padrões sendo apresentado como agente moluscicida frente ao caramujo transmissor da esquistossomose.

A utilização de produtos naturais com propriedade moluscicida, têm sido interesse de pesquisadores nos últimos tempos. Nesse contexto, os OE's se destacam por serem uma alternativa mais eficaz, menos agressiva e de baixo custo para o controle da esquistossomose, quando comparados aos moluscicidas sintéticos (Simões et al., 2016).

O potencial biológico moluscicida de espécies vegetais, podem estar relacionados aos diversos metabólicos presentes nas plantas, como flavonoides, taninos, saponinas, terpenoides, dentre outros. No entanto, apesar de vários estudos com potenciais moluscicidas naturais, há poucos relatos sobre seus metabólicos ativos. Assim, é necessário determinar e entender a interação desses compostos com a ação biológica em caramujos, a fim de obter moluscicidas naturais mais ativos, biodegradáveis e seletivos (Cantanhede et al., 2010; Matos Rocha, 2013).

\section{Considerações Finais}

Por fim, através dos resultados obtidos, o óleo essencial de Origanum vulgare é constituído por Timol (72,14\%) e pcimeno $(12,25 \%)$ e atribuiu-se a eles as atividades biológicas moluscicida, apresentando ação moluscicida frente a $B$. glabrata, com a $\mathrm{CL}_{50}$ de 26,55, incentivando assim, sua aplicação para atividades biológicas moluscicida.

\section{Referências}

Blank, D. E., Alves, G. H., Freitag, R. A., Correa, R. A., de Oliveira Hubner, S., \& Cleff, M. B. (2016). Composição química e citotoxicidade de Origanum vulgare L. E Rosmarinus officinalis L. Science And Animal Health, 4(2), 117-130.

Borges, A. M., Pereira, J., Cardoso, M. G., Alves, J. A., \& Lucena, E. M. P. (2012). Determinação de óleos essenciais de alfavaca (Ocimum gratissimum L.), orégano (Origanum vulgare L.) e tomilho (Thymus vulgaris L.). Revista Brasileira de Plantas Medicinais, 14, 656-665.

Brasil. Ministério da Saúde. Agência Nacional de Vigilância Sanitária. Resolução RDC n 26, de 13 de maio de 2014 . Dispõe sobre o registro de medicamentos fitoterápicos e o registro e a notificação de produtos tradicionais fitoterápicos. Diário Oficial [da] República Federativa do Brasil, Poder executivo, Brasília, DF. 
Cantanhede, S. P. D., Marques, A. D. M., Silva-Souza, N., \& Valverde, A. L. (2010). Atividade moluscicida de plantas: uma alternativa profilática. Revista Brasileira de Farmacognosia, 20, 282-288.

Cunha, A. P., Nogueira, M. T., \& Roque, O. R. (2012). Plantas aromáticas e óleos essenciais: composição e aplicações. Fundação Calouste Gulbenkian, 678. de Souza Luna, J. (2006). Estudo de plantas bioativas.

Everton, G. O., Teles, A. M., Mouchrek, A. N., \& Victor Filho, E. M. (2018). Aplicação do Óleo Essencial de Pimenta Dioica Lindl. como Moluscicida Frente ao Caramujo Transmissor da Esquistossomose. Revista Processos Químicos, 12(23), 85-93.

Gomes, P. R. B., Reis, J. B., da Silva, J. C., de Oliveira, R. W. S., de Paula, M. D. L., Louzeiro, H. C., \& Fontenele, M. A. (2019). Avaliação da toxicidade e atividade moluscicida do óleo essencial Cinnamomum zeylanicum Blume contra o caramujo Biomphalaria glabrata (Say, 1818). Revista Colombiana de Ciencias Químico-Farmacéuticas, 48(1), 122-127.

Júnior, P. S. S., Everton, G. O., Rosa, P. V. S., dos Santos Souza, L., de Assunção Conceição, F. O. V., da Cruz Soares, L. B., \& Mouchrek Filho, V. E. (2020). Atividade larvicida do óleo essencial de Alpinia zerumbetfrente as larvas do mosquito Aedes aegypti. Research, Society and Development, 9(8), e194985578-e194985578.

Khan, M., Khan, S. T., Khan, N. A., Mahmood, A., Al-Kedhairy, A. A., \& Alkhathlan, H. Z. (2018). The composition of the essential oil and aqueous distillate of Origanum vulgare L. growing in Saudi Arabia and evaluation of their antibacterial activity. Arabian journal of chemistry, 11(8), 1189-1200.

Leja, K. B., \& Czaczyk, K. (2016). The industrial potential of herbs and spices? A mini review. Acta Scientiarum Polonorum Technologia Alimentaria, 15(4), 353-365.

Lubbe, A., \& Verpoorte, R. (2011). Cultivation of medicinal and aromatic plants for specialty industrial materials. Industrial Crops and Products, 34(1), 785801.

McCreesh, N., \& Booth, M. (2014). The effect of simulating different intermediate host snail species on the link between water temperature and schistosomiasis risk. PloS one, 9(7), e87892.

Miranda, C. A. S. F., Cardoso, M. D. G., Batista, L. R., Rodrigues, L. M. A., \& Figueiredo, A. C. D. S. (2016). Óleos essenciais de folhas de diversas espécies: propriedades antioxidantes e antibacterianas no crescimento espécies patogênicas1. Revista Ciência Agronômica, 47, $213-220$.

La Pergola, A., Restuccia, C., Napoli, E., Bella, S., Brighina, S., Russo, A., \& Suma, P. (2017). Commercial and wild Sicilian Origanum vulgare essential oils: chemical composition, antimicrobial activity and repellent effects. Journal of EssEntial oil rEsEarch, 29(6), 451-460.

Organization, W. H. (1983). Report of the Scientific working Group on Plant Molluscicide \& Guidelines for evaluation of plant molluscicides. TDR/SCHSWG (4)/83.3. Unpublished.

Ribeiro, S. M., Bonilla, O. H., \& Lucena, E. M. P. (2018). Influência da sazonalidade e do ciclo circadiano no rendimento e composição química dos óleos essenciais de Croton spp. da Caatinga. Iheringia, Série Botânica, 73(1), 31-38.

Ribeiro, E. C. G. (2016). Atividade moluscicida de óleos essenciais de plantas aromáticas da região Amazônica maranhense.

Rocha, T. J. M., do Nascimento Filho, B. P., da Rocha Noé, B. D., Júnior, C. P. V., Costa, G. N., Aragão, M. B., \& dos Santos, A. F. (2013). Estudo do efeito moluscicida de espécies vegetais em embriões e caramujos adultos de Biomphalaria glabrata Say, 1818 (Gastropoda, Planorbidae). Revista de Patologia Tropical/Journal of Tropical Pathology, 42(2).

Sá, J., Siqueira, W., Silva, H., Calazans, R., Morais, V., Santos, M., \& Melo, A. (2017). Avaliação da atividade moluscicida do extrato de Anadenanthera colubrina sobre caramujos adultos e embriões da espécie Biomphalaria glabrata. Blucher Biophysics Proceedings, 1(1), 26-29.

Sarikurkcu, C., Zengin, G., Oskay, M., Uysal, S., Ceylan, R., \& Aktumsek, A. (2015). Composition, antioxidant, antimicrobial and enzyme inhibition activities of two Origanum vulgare subspecies (subsp. vulgare and subsp. hirtum) essential oils. Industrial Crops and Products, 70, 178-184.

Silva, L. C. P., de Lima Silva, J. V., dos Santos Melo, T. S., da Silva, D. R. S., Costa, D. G., \& de Vasconcelos, T. C. L. (2020). Análise in vitro da atividade antimicrobiana do extrato de Vaccinium macrocarpon (Cranberry) e óleo essencial de Origanum vulgare (Orégano) frente à cepa de Escherichia coli. Brazilian Journal of Development, 6(9), 70057-70069.

Silva, T. K., Borges, B. G., de Freitas, A. S., de Oliveira Soares, M. G., Freitas, E. J., Alcantra, E., \& Figueiredo, J. R. M. (2019). Atividade antifúngica in vitro de própolis sobre Colletotrichum spp. do abacate. Revista da Universidade Vale do Rio Verde, 16(3).

Simões, C. M. O., Schenkel, E. P., de Mello, J. C. P., Mentz, L. A., \& Petrovick, P. R. (2016). Farmacognosia: do produto natural ao medicamento. Artmed Editora. 